\title{
Uncertainties in Plant Optimum Definition
}

Milan JAVŮREK (CZ) milan.javurek@upce.cz

Ivan TAUFER (CZ) ivan.taufer@upce.cz

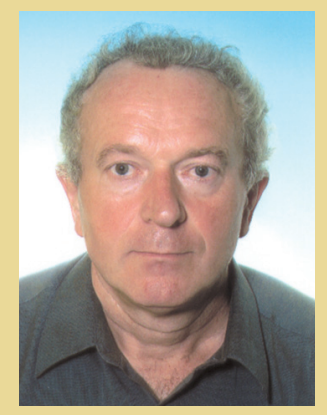

\section{ABSTRACT}

The article presents a method of simulated determination of the optimum regime in a reactor in which a competitive consecutive reaction $A \rightarrow B \rightarrow C$ takes place. The algorithm of optimization method of Kiefer-Johnson is described (method with Fibonacci numbers using), and results of experimental determination of the optimum are given. Also described are the methods of determination of limit values of confidence intervals, and the confidence intervals - uncertainty of "measured" values are evaluated from experimental data. Good accordance between the experimental results and theoretical presumptions is stated.

\section{KEY WORDS}

technological process, optimization, uncertainty of measurement, confidence interval.

\section{INTRODUCTION}

The basic requirement to be met in running any technological process is that of its effective operation, which consists in ensuring the minimum or the maximum values of selected parameters $y$, e.g. the minimum operation costs, the maximum profit, the maximum yield etc. Such a regime which exhibits the extreme values of these parameters is referred to as the optimum regime, and the controlling parameters $u$, which describe this regime are called the optimum parameters. However, in this case it must be taken into account that any technological process is a dynamic process in which a modification of a control parameter is followed by its stabilization after a certain time period only. Moreover, the process can also be affected by external effects and/or errors in measurement, which are summarily denoted as disorders $v$, which distort the setting or evaluation of the optimum regime. A flow chart of such process is given in Fig. 1.

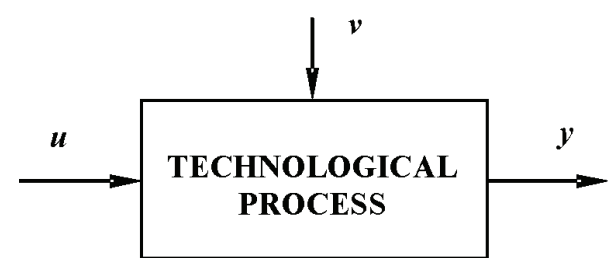

Fig. 1: Scheme of technological process.

\section{OPTIMIZATION}

For our purposes, the term optimization and/or the optimum control of technological process will mean goal-directed activity of man (operator, technologist) which under the given operational conditions (limiting parameters) ensures the best possible results of operation of the given process [2].

The aim of optimization then consists in finding such values of control quantities $u$ (the amount of staring material, temperature, pressure etc.) for which the output controlled quantities $y$ (the product quality indicators, efficiency of apparatus, energy consumption etc.) fulfill the required aspects of the optimum regime under the given technological 
conditions (e.g., the performance of apparatus, quality of starting materials and auxiliary materials etc.) and disorders $v$ (change of environment, change in quality of starting material, errors of measurements etc.). Therefore, in order to evaluate this aspect one has to define an unambiguous criterion, the optimization criterion that expresses the optimum quantitatively.

With regard to the fact that no mathematical description characterizing the relationship between the optimization criterion and its control quantities is available for a real technological process, the setting and evaluation of the optimum regime must be approached experimentally. A number of methods and algorithms can be used for this purpose.

\section{ALGORITHMS OF EXPERIMENTAL OPTI- MIZATION}

These algorithms belong into the area of the socalled non-linear programming [2], [3]. They are numerical methods of iterative nature, where the new values of independent variables $u_{k}$ in a given iteration step $\mathrm{k}$ are calculated from the values of the preceding step by adding an increment $\Delta u_{k}$. Hence, it is:

$$
u_{k}=u_{k-1}+\Delta u_{k} .
$$

It is obvious that in looking for the maximum of the given function $y(u)$ a successful step will be characterized by the following inequality:

$$
y\left(u_{k}\right)>y\left(u_{k-1}\right) .
$$

In the opposite case, the transition to state $u_{k}$ is undesirable: a new value of $\Delta u_{k}$ has to be fixed for calculation of the next value of $u_{k}$, and the calculation must be repeated. And it is just the calculation of this value $\Delta x_{k}$ which characterizes the respective method of solution. The calculation is finished, when the error of solution expressed as a difference between two consecutive vectors of control variables $u_{k}$ and $u_{k-1}$ (convergence criterion) decreases below a preset value. Then it is:

$$
|| u_{k}-u_{k-1} \|<\varepsilon .
$$

In order to demonstrate the optimisation process, we will use the method of one-parameter search, the method by Kiefer and Johnson using the Fibonacci series numbers.

\section{KIEFER-JOHNSON METHOD (METHOD USING FIBONACCI SERIES NUMBERS)}

The basic principle of the method [2], [3], [7], [8] consists in finding out the change of controlling quantity $u$ with the step $\Delta u$ in the direction of increasing value of optimisation criterion (users function) $y(u)$ according to the relationship:

$$
u_{k}=u_{k-1}+\Delta u_{k}
$$

The Fibonacci series is defined by a recurrent relationship in the form

$$
F_{k}=F_{k-1}+F_{k-2}, \quad \text { where } \quad F_{0}=F_{1}=1 .
$$

Hence, this is a series whose elements gradually increase in value. The idea of this search for extreme value then consists in the fact that the calculation of change step of independent variable $\Delta u$ uses the values of elements of Fibonacci series arranged in decreasing order. In this way, the value of this step gradually decreases during approaching the extreme value looked for. The algorithm proper reads as follows:

An auxiliary value (6) is determined for the chosen error $\varepsilon$ of solution and for the given interval of independent variable $\langle a, b\rangle$ in which the extreme value of users function should be determined

$$
M=\frac{b-a}{\varepsilon} .
$$

Using this value, one can then determine the serial number of element of the Fibonacci series so as to get

$$
F_{S-1}<M<F_{S} .
$$

Thereafter, the lowest value of step $\delta$ is calculated from the equation:

$$
\delta=\frac{b-a}{F_{S}}
$$

and then the first two values of independent variable:

$$
u_{0}=a ; \quad u_{1}=a+\delta F_{S-2}
$$

and the corresponding values of users function $y\left(u_{0}\right)$ and $y\left(u_{1}\right)$. Further values of the independent variable are then gradually calculated from the relationship:

$$
u_{i+1}=u_{i} \pm \delta F_{S-i-2} .
$$


The sign is determined from the result of preceding step in such a way that it is positive if the step is successful, i.e. if it is

$$
y\left(u_{i}\right)>y\left(u_{i-1}\right)
$$

and negative in the opposite case. This ensures the procedure of looking for the minimum in the direction of increasing users function. The calculation is finished after exhausting all the values of elements of the Fibonacci series.

\section{TECHNOLOGICAL PROCESS}

The course of optimization of technological process will be demonstrated on a model of a continuousflow perfectly stirred chemical reactor in which a competitive consecutive reaction $A \rightarrow B \rightarrow C$ takes place [8]. This reaction can be mathematically described as follows:

$$
x_{B}=\frac{\tau k_{1}}{\left(1+\tau k_{1}\right)\left(1+\tau k_{2}\right)} x_{\mathcal{A}_{0}},
$$

where $k_{1}, k_{2}$ are reaction rates from Arrhenius relationship:

$$
k=\mathcal{A} e^{-\frac{E}{R T}},
$$

$\tau$ is the retention time of reaction mixture in the reactor

$$
\tau=\frac{V}{Q}
$$

$x_{\mathcal{A}_{0}}-$ initial concentration of substance $A$,

$\mathcal{A}$ - frequency factor,

$E$ - activation energy,

$R$ - universal gas constant,

$T$ - reaction temperature,

$V$ - volume of reactor,

$Q$ - flow rate of reaction mixture through reactor.

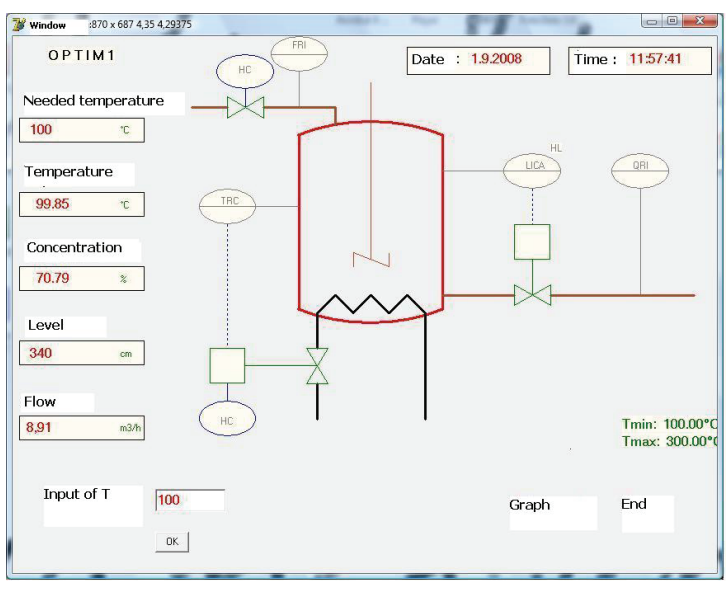

Fig. 2: Technological scheme of reactor.

The technological scheme of this continuousflow perfectly stirred chemical reactor is presented in Fig. 2. The reactor is fed with reaction mixture of the concentration $x_{\mathcal{A}_{0}}$ at the flow rate $Q$. The constant volume $V$ of reaction mixture in reactor, expressed by the level $h$, is maintained by regulating the outflow of reaction mixture from the reactor. The required temperature $T$ of reaction mixture in the reactor is regulated by flow rate of coolant in cooling pipelines. The aim of the optimum control is to obtain the maximum yield from the reaction (expressed by concentration of component $B$ ) by changing the temperature in reactor.

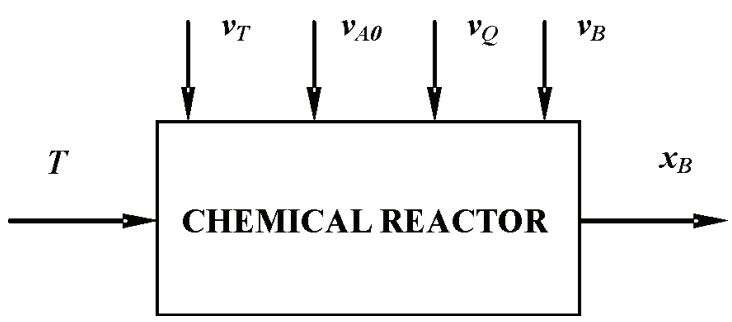

Fig. 3: Scheme of chemical reactor.

The above-given equation (12) represents a oneparameter non-linear dependence of concentration of component $B$ upon the temperature of reaction mixture in the reactor. In order to approach real conditions, all parameters of equation (12) (inlet concentration of component $A$, flow rate of reaction mixture through the reactor, the temperature of the reaction mixture, and the outlet concentration of component $B$ ) are loaded with normal-distribution random error, which should reflect the unstable val- 
ues (disorders) of these parameters and the uncertainty connected with their measurement. Moreover, dynamics of this process is simulated by introducing the 1st-order transfer function. Flow chart of this process is presented in Fig. 3.

\section{EXPERIMENT}

The following experimental conditions were chosen:

a) extent of experiment: $u_{\min }=T_{\min }=100^{\circ} \mathrm{C}$; $u_{\max }=T_{\max }=180^{\circ} \mathrm{C}$

b) convergence criterion $\varepsilon=0,5^{\circ} \mathrm{C}$.

Table 1: Results of experiment.

\begin{tabular}{|c|c|c|c|}
\hline $\mathbf{k}$ & $\mathbf{T}_{\mathbf{r}}{ }^{\circ} \mathbf{C}$ & $\mathbf{T}^{\circ} \mathbf{C}$ & $\mathbf{x}_{\mathbf{B}} \%$ \\
\hline \hline 1 & 100,00 & 101,7 & 71,83 \\
\hline 2 & 176,39 & 175,5 & 66,79 \\
\hline 3 & 129,18 & 131,3 & 83,67 \\
\hline 4 & 100,00 & 100,1 & 70,06 \\
\hline 5 & 118,03 & 119,0 & 81,16 \\
\hline 6 & 129,18 & 128,7 & 82,57 \\
\hline 7 & 136,07 & 135,2 & 83,34 \\
\hline 8 & 140,33 & 139,9 & 83,65 \\
\hline 9 & 137,70 & 139,5 & 83,63 \\
\hline 10 & 136,07 & 137,0 & 82,80 \\
\hline 11 & 135,08 & 133,6 & 82,64 \\
\hline 12 & 134,43 & 134,3 & 83,69 \\
\hline 13 & 134,10 & 133,0 & 82,52 \\
\hline
\end{tabular}

The results of experiment - search for the optimum by the Kiefer-Johnson method are presented in Table 1 , where $T_{r}$ is the required temperature value calculated by means of the above-given algorithm, $T$ is the "measured" temperature, and $x_{B}$ is again the "measured" concentration of component $B$. The optimization process was finished after the 13th step of experiment, when the absolute value of difference between two consecutive temperature values decreased below the value of error $\varepsilon$ of the solution. The optimum values obtained are $T_{\text {opt }}=134,3^{\circ} \mathrm{C}$ and the corresponding concentration of component $B, x_{B}=83,69 \%$.

A graphical representation of this search for the optimum is presented in Fig. 4, where the gradually "measured" concentration values $x_{B}$ of component $B$ are plotted against the "measured" temperature values $T$ in the reactor.

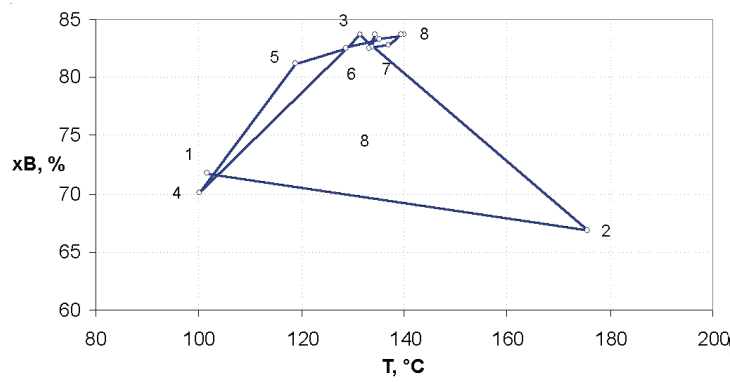

Fig. 4: Course of search for the optimum.

\section{EVALUATION OF EXPERIMENT ANTICIPATED UNCERTAINTIES OF MEA- SUREMENT RESULTS}

As we have stated above, the monitored output parameter of technological process is affected by a number of external effects that follow from instability of this process (accidental changes in parameters of starting materials and energies) and errors of measurement. Therefore, it is necessary to delimit the interval of probable values that can be assumed by the concentration $x_{B}$ of substance $B$ and temperature $T$ of reaction mixture after interferences caused by disorders of the input quantities. This standard uncertainty expressed as standard deviation [1], [4], [6] can be assessed by means of the two-point approximation method [5] according to the following (modified) relationship:

$S\left(x_{B}\right)=\sqrt{\sum_{i=1}^{m} \frac{\left[G\left[Z_{i, o p t}+S\left(Z_{i}\right)\right]+G\left[Z_{i, o p t}-S\left(Z_{i}\right)\right]\right]^{2}}{4 m}}$,

where

$G\left[Z_{i, \text { opt }} \pm S\left(Z_{i}\right)\right]$ is functional dependence (4), whose values are calculated gradually for individual limit values of monitored input quantities, 
which are given by adding their standard uncertainties to the optimum value or by subtracting them from it,

$m$ - number of the disorder quantities monitored.

Moreover, its is possible to calculate extended uncertainty as reliability interval for the given significance level [1] which will express the whole possible extent of probable values of the outlet concentration $x_{B}$

$$
S_{e}\left(x_{B}\right)=t_{1-\alpha, v} S\left(x_{b}\right),
$$

where

$t_{1-\alpha, v}$ is the critical value of Student's distribution at the significance level $\alpha$ and with the degree of freedom $v$.

In the given reactor model these disorders were simulated as random quantities with a normal distribution and a defined extent, acting on selected parameters. We simulated the disorders with the following uncertainty extent values: for the temperature of reaction mixture in reactor $S_{T}=0.5^{\circ} \mathrm{C}$, for the flow rate of reaction mixture $S_{Q}=0,1 \mathrm{~m}^{3} \mathrm{~h}^{-1}$, for the inlet concentration $S_{x_{\mathcal{A}_{0}}}=1 \%$, and for the outlet concentration $S_{x_{B}}=0,5 \%$.

After that the following values were calculated for these extents of uncertainty: standard uncertainty $S_{x_{B}}=0,49 \%$, extended uncertainty at the $5 \%$ significance level $S_{x_{B}}=1,04 \%$, and the reliability intervals $x_{B_{\min }}=82,25 \%$ and $x_{B_{\max }}=$ $84,32 \%$. These values correspond, according to equation (4), to the reliability interval for temperature as follows: $T_{\min }=124,2^{\circ} \mathrm{C}$ and $T_{\max }=$ $144,4^{\circ} \mathrm{C}$.

\section{STATISTICAL EVALUATION OF EXPERI- MENTAL RESULTS}

The above-obtained results represent the maximum possible extents based on limit values of the disorder quantities. Therefore, for evaluation of real results of "measurements" and their comparison with the above-calculated limit values it is necessary to submit the experimental data to subsequent statistical evaluation. For this purpose we used the classical regression analysis, which allows, inter alia, calculation of residual variance as a measure of variance of experimental values around the regression function $\hat{y}=f(x)$ by means of the following general relationship

$$
S_{r}=\sqrt{\frac{\sum_{i=1}^{N}\left(y_{i}-\hat{y}_{i}\right)^{2}}{N-1}},
$$

where

$y_{i}-$ the experimental results,

$\hat{y}_{i}$ - the corresponding values calculated for respective independent variables $x$,

$N-$ the number of experiments.

For obtaining the standard uncertainty and subsequently the extended uncertainty of the reaction mixture temperature, we will start from the presumption of validity of linear dependence between the experimentally found temperature and the desirable one. Then it will be:

$$
S_{r}(T)=\sqrt{\frac{\sum_{i=1}^{N}\left(T_{z, i}-T_{i}\right)^{2}}{N-1}},
$$

In this way calculated was the standard uncertainty $S_{r}(T)=1,3^{\circ} \mathrm{C}$ and the corresponding extended uncertainty at the $5 \%$ significance level $S_{e}(T)=3,0^{\circ} \mathrm{C}$, which can be used for definition of reliability interval.

The dependence of concentration of the outlet products, $x_{B}$, upon temperature $T$ was approximated by the 2 nd order regression polynomial, and the obtained relationship reads as follows:

$$
x_{B}=107,00+2,7998 T-0,0103 T^{2}
$$

the correlation coefficient being $r=0,9935$, which represents a distinct agreement between experiment and the regression dependence. The corresponding residual variance expressing the standard uncertainty is $S_{r}\left(x_{B}\right)=0,58 \%$, and the reliability interval at the $5 \%$ significance level is $S_{e}\left(x_{B}\right)=1,04 \%$. 


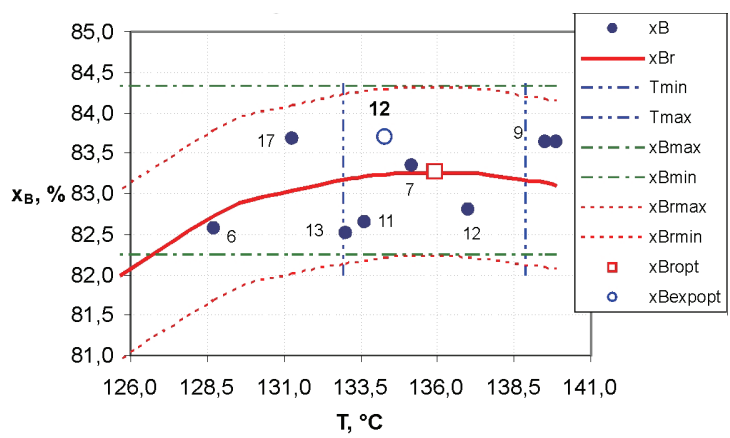

Fig. 5: Reliability intervals.

All the results obtained are summarily graphically presented in Fig. 5.

A blue full circle with serial number of experiment denotes the "measured" concentration points of monitored substance $B$, and the blue empty circle with serial number 19 denotes the "measured" optimum concentration value $x_{B}$. The green horizontal dot-and-dash straight lines denote the extended uncertainty of the limit values of outlet concentration. The extended limit uncertainty values for temperature fall outside of the diagram; therefore, they are not represented. The red solid curve depicts a part of the regression dependence $\hat{x}_{B}=f(T)$ in the vicinity of the optimum, and the red empty square on it denotes its optimum value. The red dotted curves express the uncertainty of experimental concentration values. and the blue vertical dot-anddot-and-dash straight lines express the uncertainty of experimental temperature values.

The above-mentioned tasks were solved with the use of EXCEL program and its tools: Solver, Goal Seek and Add Trendline.

\section{CONCLUSION}

When looking for the optimum conditions of a process in a reactor, one cannot limit oneself to mere expressing the monitored quantities by a single value. The quantities monitored are not only affected by disorders (external effects as well as instability in quality of starting materials) but the measurements are also loaded with uncertainty of measurement; their stabilization is a dynamic process, and reading of values of the measured quantities is also affected by subjective factors. These effects have to be taken into account, and the optimum values of quantities have to be expressed in the form of intervals in which the reactor regime can be considered to be optimal.

The result will be acceptable only in the case when the values of quantities ensuring the optimum operational regime and their respective uncertainties lie inside of the delimited area.

In our case, this interval was determined with the use of the two-point approximation method and method of determination of residual variance around the regression curve fitted to the measured points.

On the basis of the results obtained it can be stated that the optimum regime determined experimentally in the described way lies within the range of presumed reliability intervals, and the experimental results do not deviate from the limits of the found uncertainties of measurement.

\section{ACKNOWLEDGEMENT}

The problem was dealt with in the framework of research project MŠM 0021627505 "Control, optimizing and diagnostics of complex systems".

\section{REFERENCES}

[1] Chudý V., Palenčár R., Kureková E., Halaj M., Measurements of Technological Quantities (in Slovak), Bratislava, Slovak Technological University Bratislava, 1999, 688 p., ISBN 80-227-1275-2, http://www.kam.sjf.stuba.sk/katedra/publikacie/edutrac/mtv/ucebnica/obsah.htm.

[2] Drábek O., Taufer I., Automated Control Systems of Technological Processes (in Czech), Pardubice, VŠCHT Pardubice, 1990, 90 p., ISBN 80-85113-16-3.

[3] Hrubina K., Zoebel D., Heilioe M., Capel M.I., Taufer I., Krejčí S., Bradley S., Hrehová S., Šebej P., Mathematical Modelling of Technological Processes, Prešov, Faculty of Manufacturing Technologies, Technical University Košice, 2001, 216 p., ISBN 80-88941-18-0.

[4] Kubanová J., Statistical Methods for Economical and Technological Practice (in Czech), Bratislava, STATIS, 2004, 249 p., ISBN 8085659-37-9. 
[5] Meloun M., Militký J., Statistical Analysis of Experimental Data (in Czech), Prague, Academia, 2004, 953 p., ISBN 80-200-12540 .

[6] Olehla M., Věchet V., Olehla J., Dealing with Problems of Mathematical Statistics in FORTRAN Language, Prague, NADAS, 1982, 368 p., OD-31-035-82-01-13.

[7] Taufer I., Kotyk J., Hrubina K., Taufer J., Algorithms and Algorithmization, Development

Diagrams, Collection of Exercises with Key (in Czech), Pardubice, University of Pardubice, 2009, 94 p., ISBN 978-80-7395-182-5.

[8] Taufer I., Krejčí S., Javůrek M., Laboratory Exercises OPTIMIZATION, Methods of NonLinear Programming (in Czech), Pardubice, Institute of Chemical Technology, 1993.

[9] Scales L.E., Introduction to Non-linear Optimization, Sutton, Surey, MacMillan Publishers Ltd., 1985, 243 p., ISBN 0-333-32553-2.

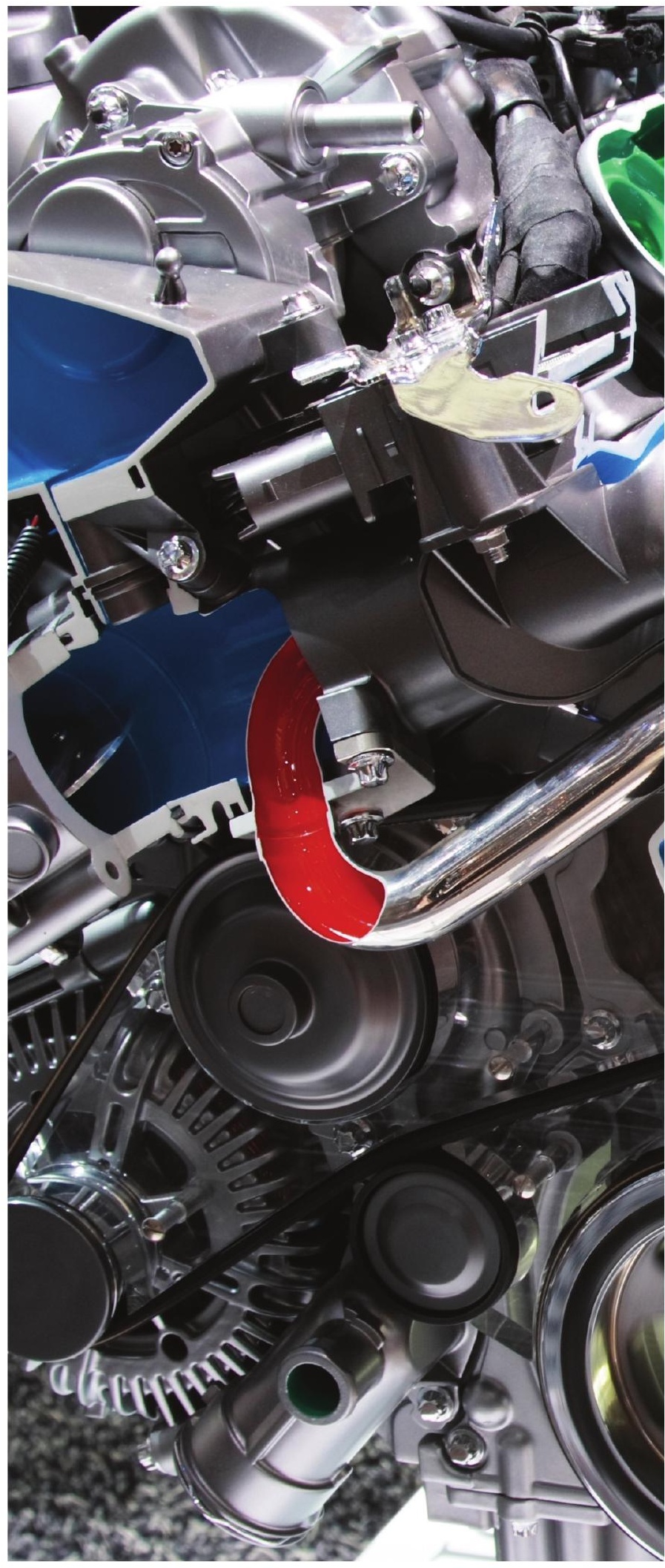




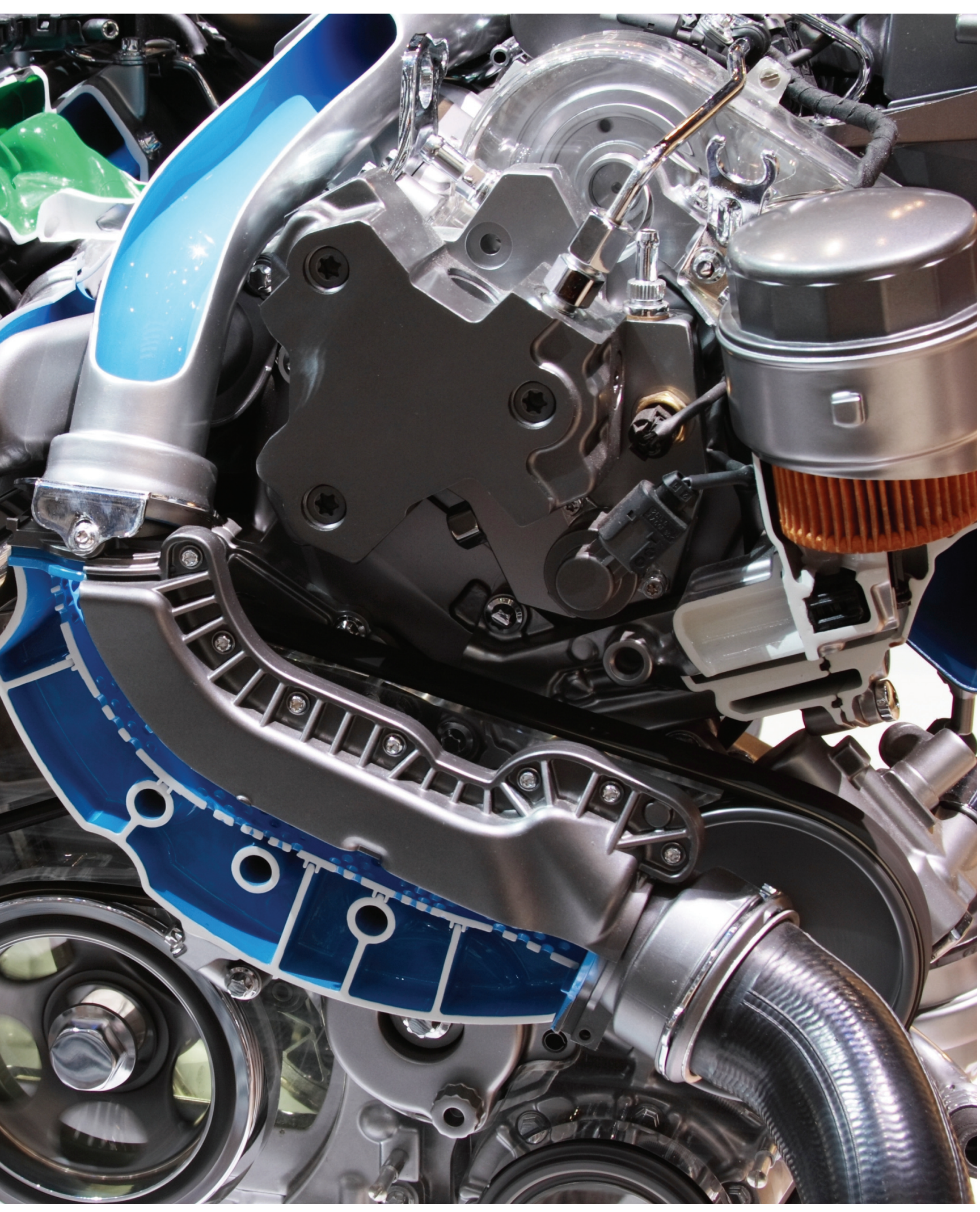

